Military Technical College Kobry El-Kobba Cairo, Egypt

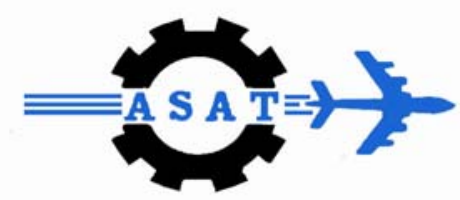

12-th International Conference on Aerospace Sciences \& Aviation Technology

\title{
SIMPLIFIED VARIATIONAL APPROACH \\ FOR ANALYSIS OF THICK ORTHOTROPIC LAMINATED PLATES: 1- CYLINDRICAL BENDING
}

\author{
M. Taha Tmerek ${ }^{\star}$, E. E. El-Soaaly ${ }^{\star *}$, M. M. El-Nomrossy ${ }^{\star}$, and A. A.Istafanous ${ }^{\star}$
}

\begin{abstract}
Reissner's, stress-based, shear deformation plate theory is chosen to approximate the stress field for balanced symmetric laminates. The longitudinal stresses are assumed to vary linearly along the plate thickness. In fact, we may view the purpose of this work as an examination of effectiveness of mathematical laminate models in which the response is defined in terms of force and moment resultants. Average stiffness moduli are considered to characterize the laminate properties. The accuracy and the range of application of the present approach are proved for laminated plate:1- in cylindrical bending and 2-simplly-supported with different thickness, for which elasticity solutions exist. The paper presents the first step of validation of the developed theory. The cylindrical bending of symmetric cross-ply laminated plates subjected to sinusoidal loading is investigated. Results from the present theory are compared with those from exact solutions and other known theories as well.
\end{abstract}

\section{KEY WORDS}

Laminate, Orthotropic, Plate, Cylindrical Bending, ESL, Variational, and Stress-based 


\section{INTRODUCTION}

It is well known that the advanced composite materials play more and more important role in aerospace structures. The evaluation of transverse shear and normal stress and the related effects have played an important, constant role in thick laminated composite structures. Recent interest in such evaluations is due to the use of composite materials in primary components, which are considerably thicker. Conventionally, the analysis of laminated plates is based on Equivalent Single Layer, ESL due to its simplicity and low computational cost. However, the ESL's with preassumed displacement field have a major deficiency. The continuity of the displacement field and its derivatives through the laminate thickness is in contradiction with the continuity of transverse stresses. Thus, the transverse stresses predicted are doubled value when using constitutive relations. To remove these discrepancies in ESL, the Layer Wise Models, LWM were introduced for the analysis of thick laminate. However, LWM's are computationally expensive due to the fact that the number of structural variables generally depend on the number of layers. For multi-layered laminate (use of 100 layers in aircraft structures is not unusual) the computations become tremendous task. Also, the computational costs, especially for geometrically nonlinear problems or transient analysis using the finite element method, preclude the use of such theories.

Pagano [1] assumed a stress distribution field in each layer in order to capture the Interlaminar effects. However, his theory results in a tedious mathematical model consisting of $23 \mathrm{~N}$ partial differential equations in the laminate's midplane coordinates and $7 \mathrm{~N}$ edge boundary conditions, where $N$ is the number of the layers in the laminate.

In the present work, a simplified variational ESL procedure is developed based on the same stress field as in [1]. The solution of a boundary value problem is considered to assess the validity of the presented approach. A comparison with the exact solutions and other models as well obtained for different span-to-thickness ratios indicates that the presented approach estimates the central deflection and the transverse stresses very well as compared to exact solution specially for the relatively thick laminates.

\section{THEORY}

Consider a laminated plate with m-orthotropic layers such that the various axes of material symmetry are parallel to the plate axes $X_{i}$. If the laminate is subjected to lateral load on the upper and lower faces by the two loads $q_{+}$and $q_{-}$respectively as indicated in Fig. 1, the total load $\mathrm{q}$ and the mean extensional load $\mathrm{p}$ may be written as

$$
\begin{aligned}
& \mathrm{q}=\mathrm{q}_{+}+\mathrm{q}_{-} \\
& \mathrm{p}=\left(\mathrm{q}_{+}-\mathrm{q}_{-}\right) / 2
\end{aligned}
$$

Reissner's, stress-based, shear deformation plate theory is based on a linear distribution of the inplane normal and shear stresses through the thickness. The assumed in-plane stress field is: 


$$
\sigma_{\alpha \beta}=\frac{1}{h} N_{\alpha \beta}\left(x_{1}, x_{2}\right)+\frac{12}{h^{3}} x_{3} M_{\alpha \beta}\left(x_{1}, x_{2}\right)
$$

where $\alpha, \beta=1,2$ and $\sigma_{\alpha \beta}$ is the normal and shear stresses. $\mathrm{N}_{\alpha \beta}$ and $\mathrm{M}_{\alpha \beta}$ are the membrane and bending moments respectively which are functions of the inplane coordinates $X_{1}$ and $X_{2} . X_{3}$ is the thickness coordinate and $h$ is the total thickness of the plate. This stress field is the simplest assumption consistent with realistic stress analysis [1].

The distribution of the transverse normal and shear stresses is determined from the equilibrium equations of the 3-D elasticity theory;

$$
\begin{aligned}
& \sigma_{\alpha 3,3}+\sigma_{\alpha \beta, \beta}=0 \\
& \sigma_{33,3}+\sigma_{\alpha 3, \alpha}=0
\end{aligned}
$$

Equilibrium of plate element is governed by overall equations containing resultant axial force, $\mathrm{N}$, shear force, $\mathrm{S}$, and bending moment, $\mathrm{M}$, Fig.1. The equilibrium equations are [2]:

$$
\begin{aligned}
& \mathrm{N}_{\alpha \beta, \beta}=0 \\
& \mathrm{M}_{\alpha \beta, \beta}-\mathrm{S}_{\alpha}=0 \\
& \mathrm{~S}_{\alpha, \alpha}+\mathrm{q}=0
\end{aligned}
$$

Using these equilibrium equations, and satisfying boundary conditions at $X_{3}= \pm \mathrm{h} / 2$ (top and bottom surfaces); we have

$$
\begin{aligned}
& \sigma_{\alpha 3}=\frac{3}{2 h}\left(1-4 \frac{x_{3}^{2}}{h^{2}}\right) S_{\alpha}\left(x_{1}, x_{2}\right) \\
& \sigma_{33}=p\left(x_{1}, x_{2}\right)+\frac{1}{2}\left(3 \frac{x_{3}}{h}-4 \frac{x_{3}^{3}}{h^{3}}\right) q\left(x_{1}, x_{2}\right)
\end{aligned}
$$

It should be noticed that the transverse shear stresses, obtained from the equilibrium equations, are parabolicly distributed through the thickness. Also, when we have surface traction free, $\sigma_{33}$ vanishes.

\section{Variational Principle for Laminates}

We apply variational analysis, based on Reissner's theory, on the assumed stress field (2) and (5). This leads to the following constitutive relations [3], which are organized to suit the orthotropic laminate:

$$
\begin{aligned}
& \mathrm{N}_{\alpha \beta}=\mathrm{h}\left(\overline{\mathrm{Q}}_{\alpha \beta \gamma \delta} \mathrm{u}_{\gamma, \delta}^{\mathrm{o}}+\overline{\mathrm{B}}_{\alpha \beta} \mathrm{p}\right) \\
& \mathrm{M}_{\alpha \beta}=\frac{\mathrm{h}^{3}}{12}\left(\overline{\mathrm{Q}}_{\alpha \beta \gamma \delta} \psi^{\gamma, \delta}+\frac{6}{5 \mathrm{~h}} \overline{\mathrm{B}}_{\alpha \beta} \mathrm{q}\right) \\
& \mathrm{S}_{\alpha}=\mathrm{h} \overline{\mathrm{d}}_{\alpha \beta}\left(\psi_{\beta}+\mathrm{w}_{, \beta}\right)
\end{aligned}
$$


where, $\overline{\mathrm{d}}_{\alpha \beta}=5 \overline{\mathrm{C}}_{\alpha 3 \beta 3} / 6, \overline{\mathrm{B}}_{\alpha \beta}=\overline{\mathrm{C}}_{\alpha \beta 33} / \overline{\mathrm{C}}_{3333}$.

For orthotropic laminate, the laminate stiffness may be considered as the linear average of the ply stiffness as follow

$\overline{\mathrm{Q}}_{\alpha \beta \gamma \delta}=\frac{1}{\mathrm{~h}} \sum_{\mathrm{n}=1}^{\mathrm{m}} \mathrm{Q}_{\alpha \beta \gamma \delta}^{\mathrm{n}} \mathrm{h}_{\mathrm{n}}$; where $\mathrm{Q}_{\alpha \beta \gamma \delta}^{\mathrm{n}}$ are the laminate reduced stiffness coefficients [4] and $h_{n}$ is the thickness of the $n$-th layer or ply group.

Similarly $\bar{C}_{i j k l}=\frac{1}{h} \sum_{n=1}^{m} C_{i j k l}^{n} h_{n}$. Where $C_{i j k l}^{n}$ are the transformed elastic coefficients [4].

\section{EXAMPLE BOUNDARY VALUE PROBLEM}

Consider a laminated plate composed of an arbitrary number of orthotropic layers such that the various axes of material symmetry are parallel to the $X_{1}-X_{2}$ axes of the plate (cross-ply laminates). In addition, let us assume a state of plane strain in which the plate is of infinite length in the $\mathrm{X}_{2}$-direction, and simply supported along the edges $\left(X_{1}=0, a\right)$, with the upper surface subjected to a normal traction: $q=-q_{o} \sin \left(m \pi x_{1} / a\right)$. Thus;

$$
\begin{aligned}
& q=-q_{o} \sin \frac{\pi x_{1}}{a} \text { for } m=1 \\
& p=-\frac{1}{2} q_{o} \sin \frac{\pi x_{1}}{a}
\end{aligned}
$$

This is a classical problem often used by researchers as a bench mark. This problem has been solved (exactly) by Pagano [5] using a three-dimensional elasticity theory.

Using above conditions, the deflected surface is cylindrical, i.e.,

$$
\mathrm{u}_{2}^{\circ}=\psi_{2}=0, \mathrm{u}_{1}^{\circ}=\mathrm{u}_{1}^{\circ}\left(\mathrm{x}_{1}\right), \psi_{1}=\psi_{1}\left(\mathrm{x}_{1}\right) \text { and } \mathrm{w}=\mathrm{w}\left(\mathrm{x}_{1}\right)
$$

Starting with: $\mathrm{N}_{\alpha \beta, \beta}=0$; due to the simple support BC [6] thus: $\mathrm{N}_{12}=\mathrm{N}_{11}=0$.

Substitute in the expression (6); we have

$$
\mathrm{u}_{1}^{\mathrm{o}}=-\frac{1}{2} \frac{\overline{\mathrm{B}}_{11}}{\overline{\mathrm{Q}}_{1111}} \mathrm{q}_{\mathrm{o}} \frac{\mathrm{a}}{\pi} \cos \frac{\pi \mathrm{x}_{1}}{\mathrm{a}}
$$

Using the equilibrium equations (4); and since we have orthotropic (symmetric) laminate; thus: $\mathrm{M}_{12}=0[6]$. Also, Since our problem is cylindrical bending; $M_{22,2}=0$ (however $M_{22} \neq 0$ ); thus; $S_{2}=0$. Hence, substitute in equilibrium equations (4); we get

$$
\mathrm{S}_{1}=-\mathrm{q}_{\mathrm{o}} \frac{\mathrm{a}}{\pi} \cos \frac{\pi \mathrm{x}_{1}}{\mathrm{a}}
$$

For simple support; $M_{11 @ x_{1}=0, a}=0$, thus 


$$
\mathrm{M}_{11}=-\mathrm{q}_{\mathrm{o}} \frac{\mathrm{a}^{2}}{\pi^{2}} \sin \frac{\pi \mathrm{x}_{1}}{\mathrm{a}}
$$

Since the present problem is cylindrical bending; thus: $\psi_{2}=0$ and also, for bidirectional (cross-ply) laminate; $\overline{\mathrm{Q}}_{1112}=\overline{\mathrm{Q}}_{1121}=0$. Thus, substitute for $\mathrm{M}_{11}$ in (6), where at $\mathrm{x}_{1}=\mathrm{a} / 2 ; \psi_{1}=0$; we get

$$
\psi_{1}=\frac{\mathrm{a}}{\mathrm{h}^{3} \pi \overline{\mathrm{Q}}_{1111}} \mathrm{q}_{\mathrm{o}}\left(\frac{12 \mathrm{a}^{2}}{\pi^{2}}-\frac{6 \mathrm{~h}^{2}}{5} \overline{\mathrm{B}}_{11}\right) \cos \frac{\pi \mathrm{x}_{1}}{\mathrm{a}}
$$

Deflection In The Thickness Direction

Substituting for $\mathrm{S}_{\alpha}$ in expression (6), the out-of-plane deflection; $\mathrm{w}$ is obtained as

$$
\mathrm{w}=-\left[\frac{1}{\mathrm{~h} \overline{\mathrm{d}}_{11}}+\frac{1}{\mathrm{~h}^{3} \overline{\mathrm{Q}}_{1111}}\left(\frac{12 \mathrm{a}^{2}}{\pi^{2}}-\frac{6 \mathrm{~h}^{2}}{5} \overline{\mathrm{B}}_{11}\right)\right] \mathrm{q}_{\mathrm{o}} \frac{\mathrm{a}^{2}}{\pi^{2}} \sin \frac{\pi \mathrm{x}_{1}}{\mathrm{a}}
$$

The Longitudinal Displacement Through The Thickness Of The Laminate

It is assumed to vary linearly through the thickness,

$$
\mathrm{u}_{1}=\psi_{1} * \mathrm{x}_{3}=\mathrm{x}_{3} \frac{\mathrm{r}}{\pi \overline{\mathrm{Q}}_{1111}} \mathrm{q}_{\mathrm{o}}\left(\frac{12 \mathrm{r}^{2}}{\pi^{2}}-\frac{6}{5} \overline{\mathrm{B}}_{11}\right) \cos \frac{\pi \mathrm{x}_{1}}{\mathrm{a}}
$$

The Longitudinal Stress

It is obtained by substituting (11) into (2); thus we have

$$
\sigma_{11}=-\frac{12}{h^{3}} x_{3} q_{o} \frac{a^{2}}{\pi^{2}} \sin \frac{\pi x_{1}}{a}
$$

The Transverse Shear and Normal Stress

Substituting for $\mathrm{S}_{\alpha}$ and (7) in expression (5), we get

$$
\begin{aligned}
& \sigma_{13}=-\frac{3}{2 h}\left(1-4 \frac{\mathrm{x}_{3}^{2}}{\mathrm{~h}^{2}}\right) \mathrm{q}_{\mathrm{o}} \frac{\mathrm{a}}{\pi} \cos \frac{\pi \mathrm{x}_{1}}{\mathrm{a}} \\
& \sigma_{33}=-\frac{1}{2} \mathrm{q}_{\mathrm{o}} \sin \frac{\pi \mathrm{x}_{1}}{\mathrm{a}}-\frac{1}{2}\left(3 \frac{\mathrm{x}_{3}}{\mathrm{~h}}-4 \frac{\mathrm{x}_{3}^{3}}{\mathrm{~h}^{3}}\right) \mathrm{q}_{\mathrm{o}} \sin \frac{\pi \mathrm{x}_{1}}{\mathrm{a}}
\end{aligned}
$$

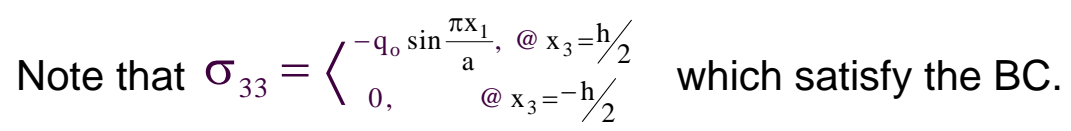




\section{NUMERICAL RESULTS AND DISCUSSION}

In order to verify the accuracy of the presented approach, 3-, 5- and 9-ply orthotropic laminates with layers of equal thickness are examined. The coordinate system of the laminate can be found in Fig. 2. All the laminates considered are symmetric with respect the central plane, with fiber orientations alternating between $0^{\circ}$ and $90^{\circ}$ with respect to the $\mathrm{X}_{1}$-axis, and the $0^{\circ}$ layers are the outer surfaces of the laminate.

Each layer is a unidireticonal fiber reinforced material with the following properties [5], which simulate a high modulus Graphite/Epoxy laminate

$$
\mathrm{E}_{\mathrm{L}}=172 \mathrm{GPa}, \mathrm{E}_{\mathrm{T}}=6.9 \mathrm{GPa}, \mathrm{G}_{\mathrm{LT}}=3.5 \mathrm{GPa}, \mathrm{G}_{\mathrm{TT}}=1.4 \mathrm{GPa}, \mathrm{v}_{\mathrm{LT}}=\mathrm{v}_{\mathrm{TT}}=0.25
$$

Where $L$ signifies the direction parallel to the fibers, $T$ is the transverse direction and $v_{\mathrm{LT}}$ is the Poisson's ratio measuring strain in the transverse direction under uniaxial normal stress in the $L$-direction.

The deflection and the normal stress in the middle of the span and the shear stress at the free edge of the laminate are of interest. The numerical results are summarized in the following sections with the normalized terms used by Pagano [5],

i.e., $\quad \bar{w}=100 E_{22} h^{3} w\left(\frac{a}{2}, 0\right) / q_{0} a^{4}, \quad \bar{u}_{1}=10 \frac{E_{22} u_{1}\left(a, x_{3}\right)}{q_{0} h}, \quad \bar{\sigma}_{11}=\frac{\sigma_{11}\left(\frac{a}{2}, x_{3}\right)}{q_{o}}, \quad \bar{\sigma}_{33}=\frac{\sigma_{33}\left(\frac{a}{2}, x_{3}\right)}{q_{o}}$, $\bar{\sigma}_{13}=\frac{\sigma_{13}\left(\mathrm{a}, \mathrm{x}_{3}\right)}{\mathrm{q}_{\mathrm{o}}}, \overline{\mathrm{X}}_{3}=\frac{\mathrm{x}_{3}}{\mathrm{~h}}, \mathrm{~S}=\frac{\mathrm{a}}{\mathrm{h}}$.

Also shown, for comparison purposes, are the results given by:

1. Higher order theory, HSDT, [7], [8], [9], [10], [13], [14]

2. First order shear deformation theory, FSDT [8],

3. First order zig-zag model [11],

4. Layerwise theory, LWM, [12] which is based on: Linear Displacement formulation; D-I and Linear Mixed formulation; M-I,

which almost represent all the models used for the analysis of laminated plates.

For all the referred references, results are due to finite element analysis and Ref[7] has closed form solution as well. Also, all these models are based on pre-assumed displacement field except [12] and [14]. Carrera [12] adopted mixed formulation in addition to a displacement field as well, while Spilker [14] used hybrid stress field.

Displacement In The Thickness Direction, $\overline{\mathrm{w}}$

The results of the displacement in the thickness direction in the middle of the laminate, at $(\mathrm{a} / 2,0)$, are listed in Table 1-3.

For a symmetric 3-ply laminate, with stacking sequence(0/90/0) is examined. Table 1. shows the values of the central deflection $\overline{\mathrm{w}}$ obtained from the different theories for $\mathrm{a}$ span-to-thickness ratio $S$ of 4 and 6(thick), 20(intermediate) and 40 (thin). 
The present approach is next tested for a symmetric 5-ply laminate. The central deflection $\overline{\mathrm{W}}$ for a span-to-thickness ratio $S$ of 4 and 6 is shown in Table 2. It is seen that the present approach has improved upon Lo, Christensen and Wu's model [13].

To further assess the accuracy of the present approach the more difficult case of a symmetric 9-ply laminate is considered. The results for the central deflection $\overline{\mathrm{w}}$ are given in Table 3 . for $S=4$ and 6 where the stability and consistency of the presented approach are observed.

\section{The Transverse Shear Stress}

Table 4. shows the values of the transverse shear stress obtained from the present approach with the elasticity, ES, and other solutions for a span-to-thickness ratio $S$ of 4 and 10(thick), 20(intermediate) and 40(thin). The results are in a good agreement with elasticity solution and in particular for the intermediate ratio 10, 20 compared to the finite element solution [9].

\section{The Transverse Normal Stress}

Table 5. shows the values of the transverse shear stress obtained from the present approach with the elasticity, HSDT and CPT solutions for a span-to-thickness ratio $\mathrm{S}=4$.

Table 6. shows the values of the transverse shear stress obtained from the present approach at the middle surface of the laminate for a span-to-thickness ratio $\mathrm{S}$ of 4(thick), 20(intermediate) and 40(thin). The results are in a good agreement with elasticity and other solutions (FEM and closed form).

\section{The Longitudinal Stress}

Table 7. shows the values of the longitudinal stress obtained from the present approach at the surface of the laminate for a span-to-thickness ratioS of 4 and 10(thick). The results are slightly better than CPT solutions, in a good agreement with the FSDT [8] for aspect ratio of 4 and comparable to the FE based on HSDT [7].

\section{CONCLUSION}

We have presented an approximate analysis, based on Reissner's stress field, for symmetric laminate consisting of orthotropic layers. Average stiffness moduli are considered to characterize the laminate properties, ESL. The accuracy of the presented approach was examined for the case of cylindrical bending of an infinitely long strip under sinusoidal loading, as numerical example, which had an elasticity solution obtained by Pagano [5]. A comparison with the exact solutions and other models as well obtained for different aspect ratios indicates that the presented 
approach estimates the central deflection and the transverse stresses very well compared to ES. While for estimating the in-plane stress, it is better than CPT and in a good agreement with FSDT proposed by Maiti and Sinha [8]. However for the longitudinal stresses, it has significant differences with the ES. This is due the assumption of continuity of the longitudinal stresses at the laminate interfaces which violates the continuity of the displacements. Therefore, the presented simplified approach yield good results for out-of-plane displacement and the transverse stresses which are responsible for the delamination failures.

\section{REFERENCES}

1. Pagano, N.J., "Stress Fields in Composite Laminates," Int. J Solids \& Structures, Vol. 14, 385-400 (1978).

2. Rehfield, L.W. and Murthy, P.L N., "Toward a New Engineering Theory of Bending: Fundamentals," AIAA J, Vol. 20, No5, 693- 699 (1982).

3. El-Soaly, E. E., "Generalization of Reissner's Theory For Thick Anisotropic Plates," $2^{\text {nd }}$ Cairo University MDP Conference, (1982).

4. Reddy, J.N., Mechanics of Laminated Composites Plates: Theory and Analysis, CRC Press, (1997).

5. Pagano, N.J., "Exact Solution For Composites Laminates in Cylindrical Bending," J Composite Materials, Vol. 3, No. 3, 398- 411, (1969).

6. Whitney, J.M., Structural Analysis of Laminated Anisotropic Plates, Technomic Publishing Co., (1987).

7. Manjunatha BS, Kant T., "Different Numerical Techniques for The Estimation of Multiaxial Stresses in Symmetric / Unsymmetric Composite and Sandwich Beams with Refined Theories." J Reinforced Plastics and Composites, Vol. 12, Jan, 237, (1993).

8. Maiti DK, Sinha PK. "Bending and free vibration analysis of shear deformable laminated composite beams by finite element method." Composite Structures 29 (1994):421-31.

9. Lee C., Liu D. "An Interlaminar stress continuity theory for laminated composite Analysis." Computer \& Structures, Vol. 42, 1, 69-78 (1992)

10. Toledano, A. and Murakami, H., "A High Order Laminated Plate Theory with improved in-plane Responses," Int J Solids Str, 23(1), pp 111-131, (1987).

11. Murakami $H$. "Laminated composite plate theory with improved in-plane responses." ASME J Appl Mech;53: 661-6, (1986).

12. Carrera E. "Evaluation of layerwise mixed theories for laminated plate analysis." AIAA Journal; 36(5):830-839, (1998).

13. Lo KH, Christensen RM, Wu EM. "A higher order theory for plate deformations, Part 2: laminated plates." ASME Trans Journal of Applied Mechanics; 44:669-76, (1977).

14. Spilker, R.L. "A Hybrid-Stress Finite-Element Formulation for Thick Multilayer Laminates," Computer \& Structures, Vol 11, 507-514, (1980). 


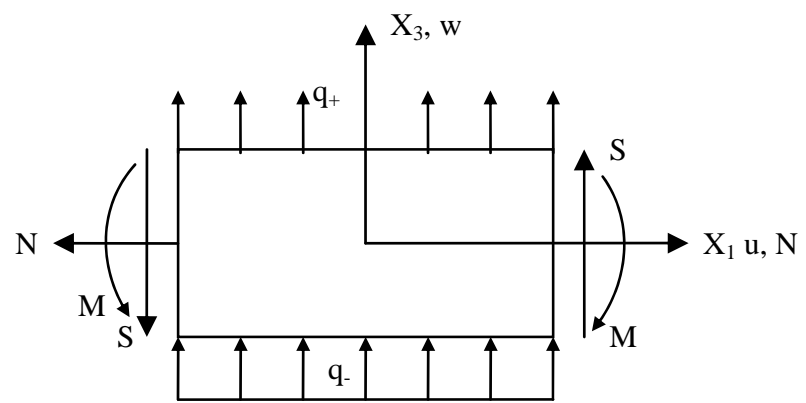

Fia.1. Thick plate loadina and sian

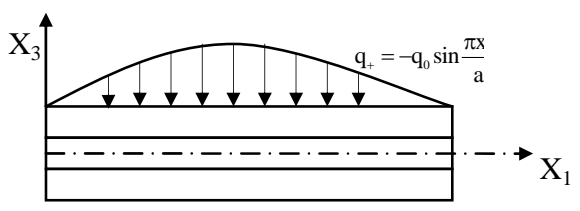

Fig. 2 Cylindrical bending of orthotropic laminated plate

Table 1. Central deflection $\overline{\mathrm{w}}(\mathrm{a} / 2,0)$ for 3-layer laminate

\begin{tabular}{|c|c|c|c|c|c|c|c|c|c|c|c|}
\hline $\mathrm{S}$ & $\mathrm{ES}^{1}$ & Present & [10] & [11] & [13] & [7] & [8] & [8] & [9] & {$[12]^{2}$} & {$[12]^{3}$} \\
\hline 4 & 2.5345 & 2.617 & 2.881 & 2.907 & 2.687 & 1.9706 & 2.7372 & 2.4146 & 2.9025 & 2.791 & 2.783 \\
\hline 6 & 1.635 & 1.565 & 1.634 & 1.636 & 1.514 & 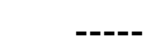 & ---- & ---- & ------ & ----- & ----- \\
\hline 10 & 0.9569 & 1.026 & ---- & ---- & ----- & 0.7492 & ---- & ----- & ----- & ----- & ----- \\
\hline 20 & 0.6172 & 0.799 & ---- & ---- & ----- & ----- & ---- & ---- & 0.6194 & ----- & ----- \\
\hline 40 & 0.5367 & 0.742 & ----- & ---- & ----- & ----- & ----- & ---- & 0.538 & ----- & ----- \\
\hline
\end{tabular}

ES, Elasticity (Exact) ${ }^{1}$ results quoted by [7], [8]\&[9] $\quad{ }^{2}$ LWM (D-I) ${ }^{3}$ LWM (M-I)

Table 2. Central deflection $\overline{\mathrm{w}}(\mathrm{a} / 2,0)$ for 5-layer laminate

\begin{tabular}{|l|l|r|c|c|c|c|c|}
\hline S & ES & Present & {$[10]$} & {$[11]^{1}$} & {$[13]^{1}$} & \multicolumn{1}{c|}{$[12]$} & \multicolumn{1}{c|}{$[12]$} \\
\hline 4 & 3.044 & 2.797 & 3.032 & 3.018 & 2.597 & 2.984 & 3.005 \\
6 & 1.721 & 1.687 & 1.716 & 1.702 & 1.507 & ----- & ----- \\
\hline
\end{tabular}

${ }^{1}$ results quoted by [10] 
Table 3. Central deflection $\overline{\mathrm{w}}(\mathrm{a} / 2,0)$ for 9-layer laminate

\begin{tabular}{|l|c|r|c|c|c|}
\hline S & ES & Present & {$[10]$} & {$[11]^{1}$} & {$[13]^{1}$} \\
\hline 4 & 3.324 & 2.929 & 3.313 & 3.231 & 2.835 \\
6 & 1.929 & 1.778 & 1.921 & 1.875 & 1.708 \\
\hline
\end{tabular}

${ }^{1}$ results quoted by [10]

Table 4. 3-ply Laminate; $\bar{\sigma}_{13}(0,0)$

\begin{tabular}{|c|r|r|c|c|c|c|r|}
\hline S & \multicolumn{1}{c|}{ ES } & Present & {$[7]$} & {$[8]^{1}$} & {$[8]^{2}$} & {$[9]^{3}$} & {$[9]^{4}$} \\
\hline 4 & 1.5974 & 1.91 & 1.782 & 1.5707 & 1.6063 & 1.4548 & 1.4244 \\
\hline 10 & 4.2347 & 4.7746 & 4.648 & ---- & ----- & ---- & ---- \\
\hline 20 & 8.749 & 9.5492 & ---- & ---- & ---- & 9.7509 & 8.7462 \\
\hline 40 & 17.634 & 19.0986 & ---- & ---- & ---- & 23.485 & 17.634 \\
\hline
\end{tabular}

${ }^{1}$ HSDT $\quad{ }^{2}$ FSDT $\quad{ }^{3}$ FEM $\quad{ }^{4}$ Closed form

Table 5. $\bar{\sigma}_{33}\left(\mathrm{a} / 2, \overline{\mathrm{X}}_{3}\right)$ for 3-ply Laminate; $(\mathrm{S}=4)$

\begin{tabular}{|c|r|c|c|c|c|c|c|c|c|c|}
\hline$\overline{\mathrm{X}}_{3}$ & -.5 & -.4 & -.3 & -.2 & -.1 & 1 & 2 & 3 & 4 & 5 \\
\hline $\mathrm{ES}$ & 0 & 0.042 & 0.145 & 0.268 & 0.395 & 0.626 & 0.737 & 0.863 & 0.953 & 1.00 \\
\hline Present & 0 & 0.028 & 0.104 & 0.216 & 0.352 & 0.648 & 0.784 & 0.896 & 0.972 & 1.00 \\
\hline$[7]$ & 0 & 0.038 & 0.138 & 0.271 & 0.414 & 0.558 & 0.701 & 0.837 & 0.946 & 1.00 \\
\hline CPT $^{1}$ & 0 & 0.263 & 0.105 & 0.216 & 0.368 & 0.658 & 0.789 & 0.9 & 0.987 & 1.00 \\
\hline \multicolumn{7}{|c|}{ 'results quoted by [7] } \\
\hline
\end{tabular}

Table 6. 3-ply Laminate; $\bar{\sigma}_{33}(\mathrm{a} / 2,0)$

\begin{tabular}{|c|c|r|r|c|}
\hline S & ES & Present & \multicolumn{1}{c|}{$[9]^{2}$} & \multicolumn{1}{c|}{$[9]^{3}$} \\
\hline 4 & 0.4988 & 0.5 & 0.5 & 0.4988 \\
\hline 20 & 0.5001 & 0.5 & 0.5018 & 0.5001 \\
\hline 40 & 0.5 & 0.5 & 0.5018 & 0.5 \\
\hline
\end{tabular}

Table 7. 3-ply Laminate; $\bar{\sigma}_{11}(\mathrm{a} / 2 \mathrm{~h} / 2)$

\begin{tabular}{|c|c|c|c|c|c|c|}
\hline $\mathrm{S}$ & ES & Present & {$[7]^{1}$} & $\mathrm{CPT}^{2}$ & {$[8]^{1}$} & {$[8]^{3}$} \\
\hline 4 & 18.41 & 9.727 & 13.89 & 9.641 & 18.43 & 10.516 \\
\hline 10 & 70.2564 & 60.7927 & 67.4 & 59.692 & ------ & ------ \\
\hline
\end{tabular}

